\title{
Numerical simulation of phosphorus release from resuspended sediment
}

\author{
Pengda Cheng ${ }^{1} \cdot$ Xinquan Wang $^{1,2} \cdot$ Chun Feng $^{1}$
}

Received: 29 April 2020 / Revised: 8 July 2020 / Accepted: 8 July 2020 / Published online: 11 November 2020

(C) The Chinese Society of Theoretical and Applied Mechanics and Springer-Verlag GmbH Germany, part of Springer Nature 2020

\begin{abstract}
One of the main issues in environmental hydraulics is pollutant release from sediments. For instance, the strong affinity between phosphorus and sediment permits most of the phosphorus to be adsorbed on the surface of the sediment particles in rivers or lakes. Post sediment resuspension, phosphorus is desorbed from the sediment to the overlying water. The release of phosphorus from the resuspended sediment is an important process in the secondary pollution of water. Herein, a coupled mechanical model of the overlying water, sediment, and pollutant was established based on the experimentally gathered data. Two types of sediment with different adsorption and desorption characteristics were selected to simulate the process of sediment resuspension and phosphorus release under different hydrodynamic conditions. The simulation results were subsequently used to analyze the relationship between the flow field characteristics and phosphorus concentration, from which the relationships between velocity, particle volume fraction, turbulent kinetic energy, total phosphorus concentration, desorbed phosphorus concentration, and time were elucidated. Based on the results, phosphorus is rapidly released into the overlying water from the resuspended sediment, and it reaches a peak value in a short duration. Unlike the release process of non-adsorption pollutants, hydrodynamic conditions and sediment properties play a crucial role in the phosphorus release process. The turbulent kinetic energy rapidly increases with the flow velocity, whereas the desorbed phosphorus concentration exhibits a certain relationship with the particle volume fraction and turbulent kinetic energy. In particular, the turbulent kinetic energy increases the desorbed phosphorus concentration per unit time. Additionally, the time taken by the total phosphorus concentration to attain its peak value is closely related to the characteristics of the flow field, whereas the amount of phosphorus is closely related to sediment properties. Post sediment resuspension, the release of phosphorus shows the characteristics of a centralized and massive release, which suggests that the total phosphorus concentration in the overlying water would change in a short duration and cause secondary pollution in the water environment.
\end{abstract}

Keywords Resuspended sediment · Adsorption · Desorption · Turbulent kinetic energy $\cdot$ Phosphorus

\section{Introduction}

Considered to be a natural complex, a water body comprises several elements: water, soluble substances, resuspended substances, aquatic organisms, and sediment [1]. Pollutants entering the water body deposit sediment that accumulates over time to form a thick sediment layer containing different pollutants, transforming the sediment into a reservoir of

Chun Feng

fengchun@imech.ac.cn

1 Institute of Mechanics, Chinese Academy of Sciences, Beijing 100190, China

2 University of Chinese Academy of Sciences, Beijing 100049, China pollutants. When the sediment is resuspended under hydrodynamic conditions, the numerous pollutants constituting the structure are released, causing secondary pollution in the water body. This internal release of the contaminated sediment becomes more pronounced when external pollution is controlled [2, 3]. Post sediment resuspension, the transport and transformation of the pollutants closely resemble sediment movement in the water [4]. On the one hand, the movement of the resuspended sediment directly affects the temporal and spatial distribution of the pollutants. On the other hand, the sediment deposition becomes a potential "source" or "sink" of the pollution mechanism, which changes under hydrodynamic disturbance and environmental conditions [5, 6]. Phosphorus, an important biogenic element in the water ecosystem, provides nutrition for algae, 
fish, and other aquatic organisms; it is one of the key factors contributing to the eutrophication of water. Two forms of phosphorus can be found in natural water: (1) dissolved phosphorus, which includes dissolved organic and inorganic phosphorus, and (2) particulate phosphorus, i.e., the type existing in phytoplankton cells or adsorbed on particulate matter. Unlike other pollutants, phosphorus in water exhibits a strong affinity with the surface of the sediment particles, which causes most of it to get adsorbed on the surface. As demonstrated in several monitoring results, the concentration of adsorbed phosphorus is much higher than that of dissolved phosphorus, rising up to as high as $90 \%$ of the total amount of phosphorus in serious cases [7]. Therefore, understanding the relationship among flow field characteristics, sediment properties (maximum adsorption capacity, adsorption strength, and water content), and phosphorus release is necessary to accurately describe the transport of phosphorus from resuspended sediment into the water environment.

Previous research on the release of pollutants from sediment primarily focused on relatively static or low-velocity water. Considering sediment to be porous media, the transport of pollutants has been systematically analyzed by coupling seepage and concentration fields. Zhu et al. [8] proposed a material exchange that mainly occurs in $5-10 \mathrm{~cm}$ of the sediment's surface layer and demonstrated that the sediment can release a considerable amount of phosphorus from pore water to the overlying water through hydrodynamic action in Taihu Lake. Wang et al. [9-12] conducted a flume experiment, based on which they formulated their release and diffusion law of sediment pollutants; this law describes the influence of sediment pollutants on water quality such as turbidity, chemical oxygen demand, and biochemical oxygen demand of the overlying water under dynamic flow conditions (different water depths and flow velocities). However, the traditional analysis of resuspended sediment has predominantly centered around the interaction between sediment and water flow $[13,14]$, and only few studies exist on the release of pollutants after sediment resuspension. The primary methods used to examine the nature of pollutant release post sediment resuspension include experimentations and monitoring, but the mechanism of hydrodynamic release requires further research. Fetters et al. [15] experimentally resuspended metal-contaminated sediments from a site in Lake DePue, Illinois (USA) and two sites in Portsmouth Naval Shipyard, Maine (USA), claiming that a short-term (4 h) sediment resuspension can mobilize metal in water, and the amount of release can be related to the laws of hydrodynamics, redox reaction, and biochemical reaction. Matisoff et al. [16] reported that severe algal blooms in Lake Winnipeg (Canada) could be attributed to an increase in watershed nutrient loading, with considerable blooms being associated with the suspended particles. However, they found that the within-lake transport and fate of this nutrient fraction as well as the importance of internal loading via resuspension are rather complex. For instance, the results of Matisoff et al.'s study indicate that up to $7 \mathrm{~cm}$ of the nutrients remain actively resuspended over a period of up to 23 years before incorporation into deeper sediments; additionally, they found that the surficial sediments in Lake Winnipeg could serve as a significant and active source of internal nutrient loading for several decades. Lepage et al. [17] investigated the impacts of the dynamics and quality of suspended particulate matter (SPM) during a flushing operation performed in 2012 on three major dams on the upper Rhône River (France). They showed that SPM concentrations during flushing reached an average that was 6-8 times higher than that observed in the flood events recorded between 2011 and 2016. The SPM contamination was generally lower during flushing than during base flow or flood; this could be because flushing only transports SPM obtained from the resuspended sediment, with no autochthonous particles or eroded soil being transported. Fang et al. [18] investigated the environmental impact of heavy metal transport and transformation in Hangzhou Bay, China, using a comprehensive model that integrates hydrodynamics, sediment, and heavy metal transport to simulate the accidental discharge of ${ }^{137} \mathrm{Cs}$. The model was also a useful tool for predicting heavy metal transport and fate. Post sediment resuspension, the phosphorus pollutants were found to originate from the dissolved and desorbed phosphorus of the sediment particles.

The increase in resuspended particle concentration is usually visible, whereas the processes of phosphorus desorption, dissolution, and release are often ignored. Based on this premise, the total concentration of phosphorus can be easily underestimated or overstated post sediment resuspension. To understand the physical process and influencing factors during phosphorus release, a coupled mechanical model of the overlying water, sediment, and pollutant is established and described herein. Moreover, the adsorption and desorption processes of sediment are explored as they are useful in elucidating the relationships among velocity, sediment volume fraction, turbulent kinetic energy, total phosphorus concentration, desorption phosphorus concentration, and time under different hydrodynamic conditions. Subsequently, the effects of the flow field and sediment characteristics on phosphorus release are analyzed post sediment resuspension.

\section{Phosphorus adsorption and desorption}

The National Standard (HJ 670-2013) states that potassium dihydrogen phosphate $\left(\mathrm{KH}_{2} \mathrm{PO}_{4}\right)$ is the standard substance for determining total phosphorus concentration. The weight of $\mathrm{KH}_{2} \mathrm{PO}_{4}$ used in the experiment was accurately measured at $4.394 \pm 0.001 \mathrm{~g}$ using an electronic analytical balance. It was dried in an oven at $110{ }^{\circ} \mathrm{C}$ for $2 \mathrm{~h}$ cooled to room tem- 


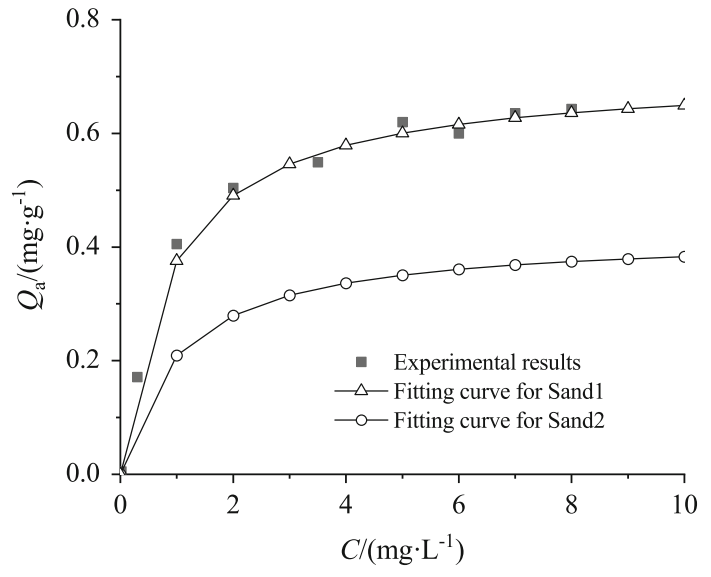

Fig. 1 Fitting curve for phosphorus isotherm adsorption

Table 1 Parameters of the Langmuir adsorption isotherm

\begin{tabular}{llll}
\hline & $Q_{\mathrm{m}}(\mathrm{mg} / \mathrm{g})$ & $K$ & Correlation coefficient \\
\hline Sand1 & 0.706 & 1.139 & 0.9668 \\
Sand2 [7] & $0.422[7]$ & $0.981[7]$ & $0.9556[7]$ \\
\hline
\end{tabular}

perature on the dryer, and then prepared into a $1000-\mathrm{mg} / \mathrm{L}$ phosphorus standard solution by adding distilled water [19].

The two types of cohesive fine sediment selected were Sand1, obtained from Suzhou River, and Sand2, from the Guanting reservoir [7]. The turbid liquid was obtained after mixing at the sediment concentration of $10 \mathrm{~g} / \mathrm{L}$, following which the $\mathrm{KH}_{2} \mathrm{PO}_{4}$ solution was added to the resulting mixture with different initial concentrations. Subsequently, the turbid liquid was shaken in a constant-temperature shaker for $24 \mathrm{~h}$. Next, the samples were centrifuged and filtered after adsorption equilibrium was attained at $25{ }^{\circ} \mathrm{C}$. Finally, the $\mathrm{PO}_{4}$ concentration in the filtrate and the adsorption amount of the unit mass sediment $(\mathrm{mg} / \mathrm{g})$ were measured. The Langmuir adsorption isotherm presented in Eq. (1) was used to fit the experimental data of the phosphorus isotherm adsorption obtained by sedimentation (Fig. 1):

$Q_{\mathrm{a}}=\frac{Q_{\mathrm{m}} K C}{1+K C}$,

where $Q_{\mathrm{a}}$ is the equilibrium adsorption capacity $(\mathrm{mg} / \mathrm{g}), Q_{\mathrm{m}}$ is the maximum adsorption capacity $(\mathrm{mg} / \mathrm{g}), C$ is the concentration of $\mathrm{P}(\mathrm{mg} / \mathrm{L})$ at equilibrium, and $K$ is the adsorption strength.

The parameters of the Langmuir adsorption isotherm could be obtained by fitting the experimental data of the phosphorus adsorption isotherm (Table 1).

In general, three types of phosphorus adsorption modes of sediment exist in any water body. They are listed as follows.
Table 2 Average velocity under different cases

\begin{tabular}{lllllll}
\hline & Case 1 & Case 2 & Case 3 & Case 4 & Case 5 & Case 6 \\
\hline $\boldsymbol{U}(\mathrm{m} / \mathrm{s})$ & 0.03 & 0.08 & 0.13 & 0.23 & 0.35 & 0.50 \\
\hline
\end{tabular}

1. Physical adsorption. Van der Waals forces acting between particles allow them to adsorb the surrounding materials, wherein high surface energy and specific surface area are conducive to physical adsorption.

2. Chemisorption. In this mode, the particles require high activation energy to establish a link with the surrounding materials through certain electrochemical actions.

3. Exchange adsorption. Also known as coordination adsorption, this process occurs when there is an exchange between $\mathrm{PO}_{4}$ and the $-\mathrm{H}_{2} \mathrm{O}$ and $-\mathrm{OH}$ groups in the particles.

The physical adsorption and desorption of phosphorus via sedimentation are often rapid and permissible under lowactivation energy. In contrast, the chemical adsorption and exchange adsorption require high activation energy because of the reactions involving chemical bond formation. Therefore, the primary form of phosphorus adsorption is the rapid physical adsorption of sediment particles.

\section{Governing equations and numerical models}

\subsection{Governing equations}

Based on previous experimental measurements, the resuspended polluted sediment mainly includes fine sediment with silt and clay, wherein $>8 \%$ particles have diameters of less than $5 \mu \mathrm{m}$. The median particle size $D_{50}$ of the polluted sediment normally ranges within $25-50 \mu \mathrm{m}$, whereas that of the surface sediment is $<30 \mu \mathrm{m}$. Herein, $D_{50}=30 \mu \mathrm{m}$ was the representative particle size selected for resuspended sediment. In the overlying water-sediment-contaminant model, the resuspended sediment is considered a suspension because of the sediment's small particle size. By analyzing a considerable amount of experimental data [20,21], the viscosity of the suspension can be expressed as a function of particle volume fraction. Therefore, the suspension can be characterized to exhibit single flow continuum with macroscopic properties such as density and viscosity.

The hypothesis of the overlying water-sediment-contaminant model is as follows.

1. The density of each phase is approximately constant.

2. Both phases share the same pressure field. 
3. The particle relaxation time is shorter than the timescale of the macroscopic flow.

Accordingly, the mixture of density and viscosity is given by

$\rho=(1-\varphi) \rho_{1}+\varphi \rho_{2}$ and

$\mu=(1-\varphi) \mu_{1}+\varphi \mu_{2}$,

where $\rho_{1}, \rho_{2}$ and $\mu_{1}, \mu_{2}$ indicate the density and viscosity properties of the fluid and particle, respectively, and $\varphi$ represents the particle volume fraction.

Considering that the suspension can be regarded as a continuous medium, its flow is governed by incompressible mass and momentum conservation equations represented by

$\nabla \cdot(\rho \boldsymbol{u})=0$ and

$\rho\left(\frac{\partial \boldsymbol{u}}{\partial t}+\boldsymbol{u} \cdot \nabla \boldsymbol{u}\right)=-\nabla p+\nabla \cdot \boldsymbol{\tau}+\rho \boldsymbol{g}+\boldsymbol{f}$,

where $\boldsymbol{u}$ is the velocity field, $\rho$ is the suspension density, $t$ is time, $p$ is the pressure field, $\tau$ is the sum of the viscous and turbulent stresses, and $\boldsymbol{g}$ is the body force per unit mass. The term $\boldsymbol{\tau}$ can be expressed as a generalized Newtonian fluid, such that $\boldsymbol{\tau}=\mu \gamma$, where $\mu$ is the viscosity and $\boldsymbol{\gamma}=\nabla \boldsymbol{u}$ $+\nabla \boldsymbol{u}^{\mathrm{T}}$. The standard $k-\varepsilon$ turbulence model was adopted for the flow-field simulations [22], and the model constants were determined from the experimental data [23]: $C_{\mu}=0.09, \sigma_{\mathrm{k}}$ $=1.0, \sigma_{\varepsilon}=1.3, C_{\varepsilon 1}=1.44$, and $C_{\varepsilon 2}=1.92$.

The transport equation for the particles in the flow is determined by

$\frac{\partial \varphi}{\partial t}+\boldsymbol{u} \cdot \nabla \varphi=-\nabla \cdot N_{\varphi}$

where $N_{\varphi}$ is the total diffusive flux of particles This expression results from two different mechanisms: first, by a flux sourced from the gradients in a particle-particle collision frequency $\left(N_{\mathrm{c}}\right)$, and second, by a flux originated from the gradients in a suspension viscosity $\left(N_{\mu}\right)$. These fluxes are determined as $N_{c}=-K_{c} a^{2} \varphi \nabla(\gamma \varphi)$ and $N_{\mu}=$ $-K_{\mu} a^{2} \frac{\gamma \varphi^{2}}{\mu} \nabla \mu$ respectively, where $K_{\mathrm{c}}$ and $K_{\mu}$ are empirical diffusive-like coefficients, $a$ is the particle radius, and $\gamma$ is the local strain rate. Of these, $N_{\mathrm{c}}$ drives the particles in the opposite direction of the gradients in the strain rate and particle concentration, and $N_{\mu}$ allows particle migration in the direction opposite to that of the gradients in suspension viscosity. Furthermore, the suspension of neutrally buoyant, non-colloidal, and spherical particles is considered, whereas additional diffusive fluxes potentially attributed to Brownian motion and sedimentation under the influence of gravity are neglected.

Accordingly, the transport equation for the contaminant concentration in the flow is given by

$\frac{\partial c}{\partial t}+\boldsymbol{u} \cdot \nabla c=D \nabla^{2} c+R$

where the relative concentration $c$ is a dimensionless value, $D$ is the diffusion coefficient, and $R$ is the $c$ generated or disappeared because of chemical reaction in unit time and unit volume, which is both the source and sink term.

The viscosity of a particle suspension is normally expressed as a function of the local particle volume fraction [24-27]. Herein, the Maron-Pierce-Quemada (MPQ) model $[24,25]$ was used for the suspension equation.

$\mu=\mu_{1}\left(1-\frac{\varphi}{\varphi_{m}}\right)^{-2}$,

where $\varphi_{\mathrm{m}}$ is the maximum-packing concentration, which for solid particles is $\sim 0.62$.

\subsection{Geometric model}

Under the influence of gravity, the flow of suspended particles was considered in the water channel. The dimension of the water channel used was the same as that used in the author's previous experiments: $0.25-\mathrm{m}$ wide and $3.0-\mathrm{m}$ long. The water and sediment depths were set as 0.1 and $0.08 \mathrm{~m}$, respectively (Fig. 2).

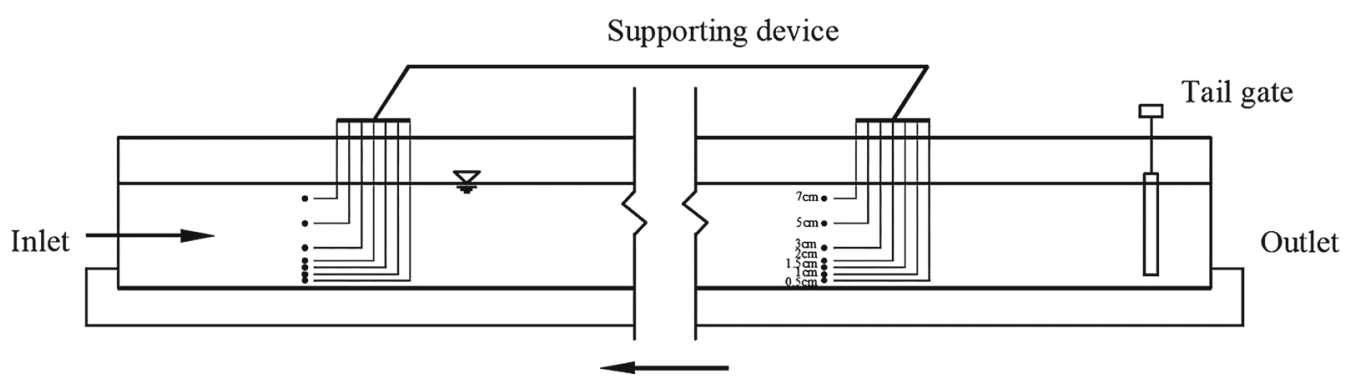

Fig. 2 Schematic of the circulating water channel 


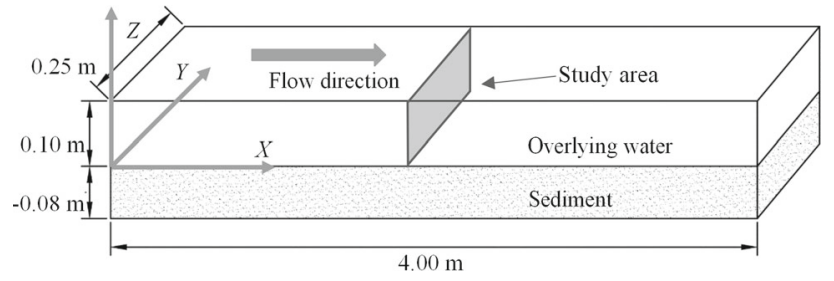

Fig. 3 Computational domain used in the simulation

Accordingly, the geometric model used in the numerical simulation was consistent with the experimental size (Fig. 3). The left side of the water-sediment interface was set as the origin of the coordinate system, whereas the study object was the middle section of the overlying water.

\subsection{Boundary and initial conditions}

In a flow field, the wall of the water channel is the no-slip boundary denoted by.

$\boldsymbol{u}=\mathbf{0}$.

In contrast, the top surface is the symmetrical boundary in which the normal velocity and shear stress are both equal to 0 , typically described as a combination of the Dirichlet and Neumann conditions.

$$
\left.\begin{array}{l}
\boldsymbol{n} \cdot \boldsymbol{u}=0 \\
K-(\boldsymbol{n} \cdot K) \boldsymbol{n}=0 \\
K=\mu\left[\nabla \boldsymbol{u}+(\nabla \boldsymbol{u})^{\mathbf{T}}\right] n
\end{array}\right\} .
$$

Further, in particles and the contaminant transport field, the wall and the top surface of the water channels are no-flux boundaries denoted by.

$$
\left.\begin{array}{l}
\boldsymbol{n} \cdot(\boldsymbol{u} c-D \nabla c)=0 \\
\boldsymbol{n} \cdot(\boldsymbol{u} \varphi-D \nabla \varphi)=0
\end{array}\right\}
$$

The periodic boundary conditions are used for the left and right boundaries in the flow field, for particles, and in the contaminant transport field, wherein the velocity, concentration, volume fraction, turbulence kinetic energy, and turbulence dissipation rate are regarded as equal.

Here, the initial velocity is 0 , and the initial pressure is related to the water depth as.

$p=p_{0}+\rho g z$,

where $p_{0}$ represents atmospheric pressure.

The sediment particle size $D_{50}$ was $0.03 \mathrm{~mm}$. The initial particle volume fraction of the sediment was $42.5 \%$ in pore water and 0 in the overlying water. Moreover, the phosphorus

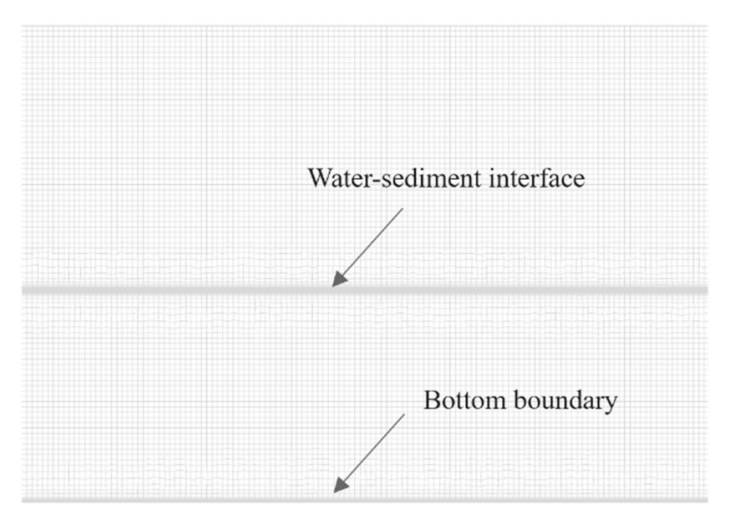

Fig. 4 Schematic of the interface grid

concentrations in pore water and in the overlying water were 10 and $0 \mathrm{mg} / \mathrm{L}$, respectively. Additionally, the phosphorus adsorption on the particles reached both the saturation and maximum adsorption capacities.

The densities of the overlying water and sediment were 997 and $2650 \mathrm{~kg} / \mathrm{m}^{3}$, respectively, and the viscosity of the overlying water was $1 \times 10^{-3} \mathrm{~Pa} \mathrm{~s}$.

Following the grid independence test, 82,800 elements of the structured grid were used in the numerical simulation, which were enhanced at the sediment-water interface and the bottom boundary. As indicated in Fig. 4, there were 16 layers at the sediment-water interface and eight layers at the bottom boundary (Fig. 4).

\subsection{Numerical method}

In this study, the finite element method (FEM) [28-30] was used to solve the flow field and the particles and contaminant transport field. A discrete version of the continuous model equations was formed using the Galerkin FEM, which begins by considering the weak form of the momentum equation derived as the product of Eq. (5) with a vector-valued test function $w \in \mathrm{H}^{1}(\Omega)^{3}$ (where $\mathrm{H}^{1}(\Omega)$ is the first Hilbertian Sobolev space) and then integrating it over the domain $\Omega$ [30]:

$$
\begin{aligned}
& \int_{\Omega} \boldsymbol{w} \cdot\left(\rho \frac{\partial \boldsymbol{u}}{\partial t}\right) \mathrm{d} V+\int_{\Omega} \boldsymbol{w} \cdot(\rho \boldsymbol{u} \cdot \nabla \boldsymbol{u}) \mathrm{d} V \\
& =-\int_{\Omega} \boldsymbol{w} \cdot(\nabla p) \mathrm{d} V+\int_{\Omega} \boldsymbol{w} \cdot(\nabla \cdot(\mu \nabla \boldsymbol{u})) \mathrm{d} V . \\
& +\int_{\Omega} \boldsymbol{w} \cdot(\rho \boldsymbol{g}) \mathrm{d} V-\int_{\Omega} \boldsymbol{w} \cdot(\boldsymbol{f}) \mathrm{d} V
\end{aligned}
$$

In this weak form, a solution to the velocity field $\boldsymbol{u}$ $\in \mathrm{H}^{1}(\Omega)^{3}$ is sought such that it is valid for all test functions $w \in \mathrm{H}^{1}(\Omega)^{3}$.

Further, discontinuous piecewise linear basis functions [31] were assigned for the velocity field such that the solution nodes were not shared between the elements as each element 
is essentially an independent problem. Within an element $e$, the test function and solution can be defined by

$$
\left.\begin{array}{l}
\boldsymbol{w}=\sum_{j=1}^{N} \psi_{j} \boldsymbol{w}_{j} \\
\boldsymbol{u}=\sum_{k=1}^{N} \psi_{k} \boldsymbol{u}_{k}
\end{array}\right\},
$$

where $N$ is the number of velocity solution nodes in the element $e$, and $\boldsymbol{w}_{\mathrm{j}}$ and $\boldsymbol{u}_{\mathrm{k}}$ are the values of the test function and the solution at nodes $j$ and $k$, respectively. The basic functions $\psi_{\mathrm{j}}$ and $\psi_{\mathrm{k}}$ are unity at nodes $j$ and $k$, whose value is zero at all other nodes. The pressure field $p$ is required when finding the coefficients $\boldsymbol{u}_{\mathrm{k}}$. Thus, $p$ can be calculated as a summation of continuous piecewise quadratic basis functions [31] defined by

$p=\sum_{l=1}^{N} \Psi_{l} p_{l}$.

where $p_{l}$ is the value of the pressure field at node $l$, and $\Psi_{l}$ is the basis function that is equal to 1 at node $l$ and 0 at all other nodes.

The discretized momentum equation yields an $N \times N$ node system of linear equations that could be assembled and solved for the vectors of unknown coefficients $\boldsymbol{u}$ and $p$ :

$\boldsymbol{M} \frac{\partial \boldsymbol{u}}{\partial t}+\boldsymbol{A} \boldsymbol{u}+\boldsymbol{K} \boldsymbol{u}+\boldsymbol{F}_{\text {left }} \boldsymbol{u}=\boldsymbol{C} p+\boldsymbol{b}+\boldsymbol{f}_{\text {right }}$.

where, $\boldsymbol{M}, \boldsymbol{A}, \boldsymbol{K}$, and $\boldsymbol{C}$ represent the mass, advection, stress, and gradient matrices, respectively. The matrix $\boldsymbol{F}_{\text {left }}$ contains the left-hand side part of the drag term, whereas the vectors $\boldsymbol{b}$ and $\boldsymbol{f}$ represent the buoyancy force and the right-hand side part of the drag term, respectively.

The solution to the discretized momentum equation must satisfy the discrete continuity equation. It can be derived from the FEM as

$$
\sum_{i=1}^{N}\left(\boldsymbol{C}_{\mathrm{i}}^{\mathrm{T}} \boldsymbol{u}-\boldsymbol{r}_{\mathrm{i}}\right)=0
$$

where the matrix $\boldsymbol{C}^{\mathrm{T}}$ acts as a divergence, and $\boldsymbol{r}$ is a surface integral term through which Dirichlet boundary conditions can be applied.

Following the pressure projection method [32, 33], the solution process begins by considering the momentum equation that has been discretized in space using the Galerkin FEM, and in time using the backward Euler method. The primary steps of the method are briefly described as follows.
1. Compute nonlinear approximations to the velocity fields using the latest (best available) tentative solutions, $\boldsymbol{u}^{\mathrm{n}+1}$ $=\theta u^{\text {tent }}+(1-\theta) \boldsymbol{u}_{\mathrm{n}}, \theta=0.5$.

2. Make a "best guess" for $p^{\text {tent }}$ by solving a pressure Poisson equation or using the most up-to-date pressure field available.

3. Solve the discretized momentum equation to obtain $\boldsymbol{u}^{*}$.

4. If $\boldsymbol{u}^{*}$ could not satisfy the continuity equation because of an unsuitable $p^{\text {tent }}$ value, then determine the pressure correction term $\Delta p$.

5. Correct $\boldsymbol{u}^{*}$ to obtain $\boldsymbol{u}^{\text {tent }}$ by substituting the recently determined $\Delta p$.

6. Use the new tentative velocities $\boldsymbol{u}^{\text {tent }}$ to provide the best available solutions for all fields in the next iteration.

As soon as the desired Picard iteration limit or convergence is reached, $\boldsymbol{u}^{\mathrm{n}+1}$ and $p^{\mathrm{n}+1}$ adopt the values of the final tentative solution, and the time step is deemed complete. The above solution procedure is repeated until a desired time limit or steady state is attained.

\section{Model validation}

To validate the proposed model, the calculated values of the non-adsorption pollutant concentration were compared with the experimental results obtained for the overlying water under different flow velocities. All non-adsorption pollutants were extracted from the dissolved pollutants in the pore water post sediment resuspension. By removing the changes in the adsorption and desorption concentration, the accuracy of the coupled mechanical model was verified more directly. Sand1 having $57.5 \%$ water content and $10 \mathrm{mg} / \mathrm{L}$ pollutant concentration was used in the water channel experiment, in which the water velocity and depth were adjusted by slowly adding water and regulating the tail gate to allow the hydrodynamic conditions to fulfill design requirements. Because of the short time of pollutant release post sediment resuspension, the measurement conditions of concentration were limited. The measured concentrations were subsequently applied at 0.15 and $0 \mathrm{~m} / \mathrm{s}$. From the result of the pollutant concentration analysis in the overlying water, the release characteristics of the non-adsorption pollutants could be obtained post sediment resuspension. In particular, the concentration of non-adsorption pollutants was calculated under a set of four different flow velocities $0.13,0.23,0.35,0.50 \mathrm{~m} / \mathrm{s}$ (Fig. 5). Under the influence of hydrodynamic conditions, the concentration in the overlying water reached equilibrium in a short duration $(<2 \mathrm{~min})$, which is consistent with the numerical simulation results [10]. Furthermore, the pollutants were released into the overlying water through molecular diffusion in static water. Comparatively, post sediment resuspension, 


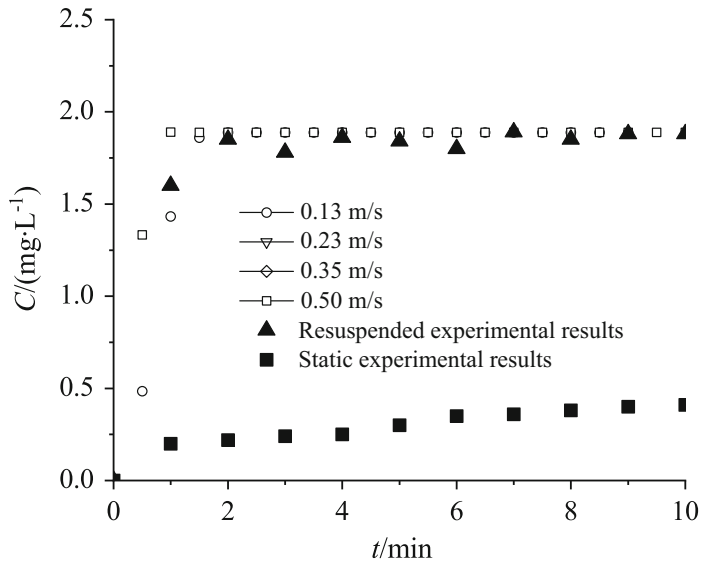

Fig. 5 Comparison of concentration of non-adsorption pollutant between simulation and experimental results

the release of pollutants in relatively-velocity water could reach five times the concentration as that in static water.

\section{Results}

In the flow field, sediment particles move in an extremely complex and random pattern. To quantitatively analyze such movement, the center profile of the overlying water was investigated using the proposed geometric model. The crosssectional average velocity $(\boldsymbol{U})$, average volume fraction $(\Phi)$, average total $\mathrm{P}$ concentration $(C)$, and average turbulent kinetic energy $(K)$ were defined by the following system of formulas,

$$
\left.\begin{array}{c}
\boldsymbol{U}=\frac{\iint_{s} \boldsymbol{u}^{2} \mathrm{~d} y \mathrm{~d} z}{\iint_{s} \boldsymbol{u} \mathrm{d} y \mathrm{~d} z} \\
\boldsymbol{\phi}=\frac{\iint_{s} \boldsymbol{u} \varphi \mathrm{d} y \mathrm{~d} z}{\boldsymbol{U} H} \\
C=\frac{\iint_{s} \boldsymbol{u} c \mathrm{~d} y \mathrm{~d} z}{\boldsymbol{U} H} \\
K=\frac{\iint_{s} \boldsymbol{u} k \mathrm{~d} y \mathrm{~d} z}{\boldsymbol{U} H}
\end{array}\right\}
$$

where $\boldsymbol{U}$ is the cross-sectional average velocity $(\mathrm{m} / \mathrm{s}), \Phi$ is the average volume fraction, $C$ is the average total $\mathrm{P}$ concentration, and $K$ is the average turbulence kinetic energy $\left(\mathrm{m}^{2} / \mathrm{s}^{2}\right)$, respectively.

Figure 6 shows the value of cross-sectional average velocity changing with time post sediment resuspension. In Cases $1-6$, the average velocities were $0.03,0.08,0.13,0.23,0.35$, and $0.50 \mathrm{~m} / \mathrm{s}$. In the initial stage of sediment resuspension, $\boldsymbol{U}$ was extremely small in all cases. Over time, it rapidly increased, reaching a stable velocity in a short period. The value of $\boldsymbol{U}$ in the aforementioned cases is listed in Table 1 .

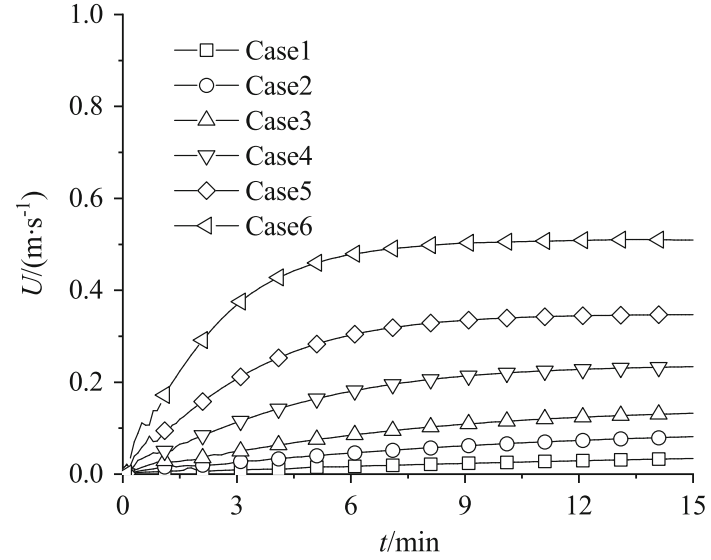

Fig. 6 Variation of average velocity over time post sediment resuspension

Figure 7 describes the vertical distribution of $\varphi$ with respect to time in Cases 3 and 4. Under different velocities, the vertical distribution of $\varphi$ was rapidly balanced with time, attaining the same stable value or uniformity in a short period ( $<3 \mathrm{~min}$ ), which is close to the experimental demonstration. Furthermore, the uniformity of $\varphi$ distribution improved with increasing velocity. For fine sediment $\left(D_{50}=0.03 \mathrm{~mm}\right)$, the spatial structure of sediment was relatively uniform and the velocity of the suspension and deposition could easily reach a balance to obtain the distribution balance of $\varphi$. Accordingly, the equilibrium could not be broken even when the hydrodynamic conditions were assumed to be constant.

The complex process of phosphorus release in sediment is mainly influenced by the interaction between the hydrodynamic characteristics and sediment properties. The resuspended sediment particles initially affect the flow characteristics, before contributing to phosphorus release. To analyze the relationship between $\Phi, K$, and time at different velocities, the data corresponding to Cases 1, 3, 4, and 5-were compared (Fig. 8). Under different velocities, the $\Phi$ of fine sediment attained equilibrium in a short duration ( $<3 \mathrm{~min}$ ), which could be attributed to the small sediment size and the difficulty of deposition. Moreover, $K$ rapidly increased with increasing $\Phi$ in the overlying water. Thus, a faster $\boldsymbol{U}$ resulted in a higher $K$ and a shorter duration for $\Phi$ to attain equilibrium. For all cases, the value of $\boldsymbol{U}$ increased by about 11.6 times, whereas the peak value of $K$ increased by more than 11 times. Additionally, as soon as the $\Phi$ of the sediment attained equilibrium, $K$ similarly tended to equilibrium. In the sediment resuspension process, the change in $K$ is closely related to the $\Phi$ in water. The fine sediment changes the flow field structure, thereby increasing $K$; consequently, additional fine particles enter the overlying water until $\Phi$ reaches equilibrium.

The monitoring results of phosphorus in natural water demonstrated that the amount of phosphorus adsorption is 

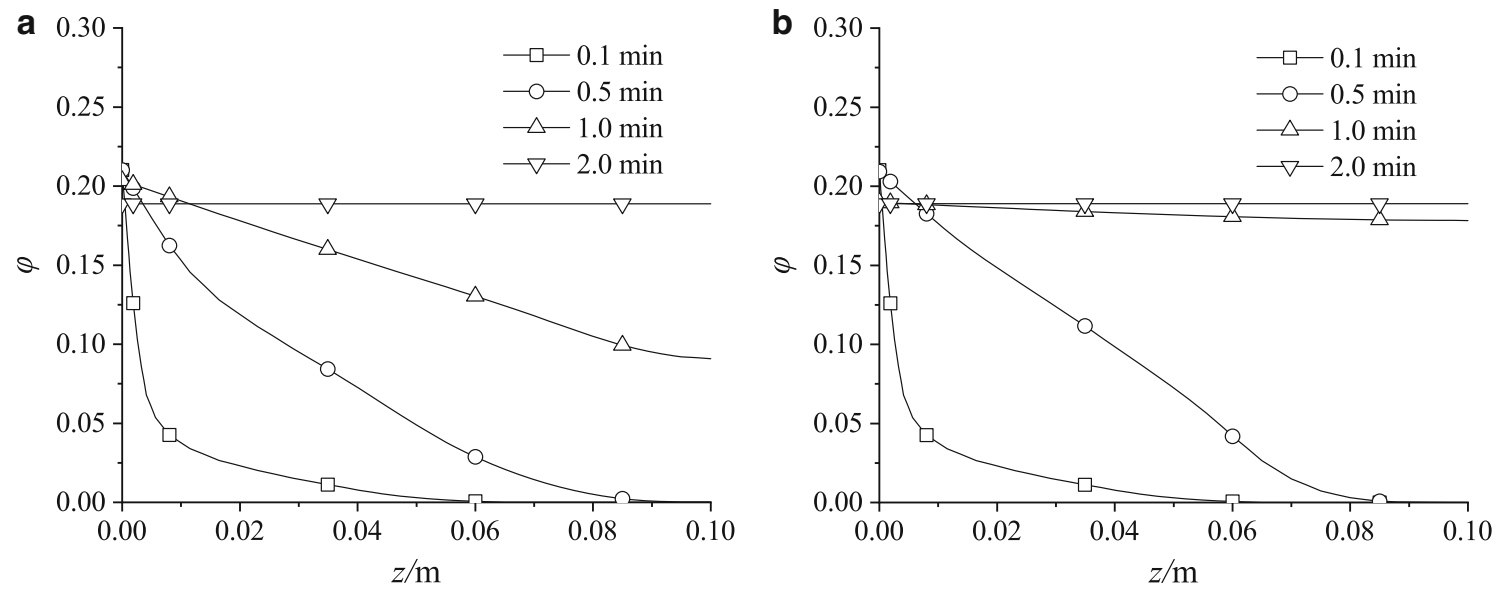

Fig. 7 Vertical distribution of $\varphi$ over time in the overlying water
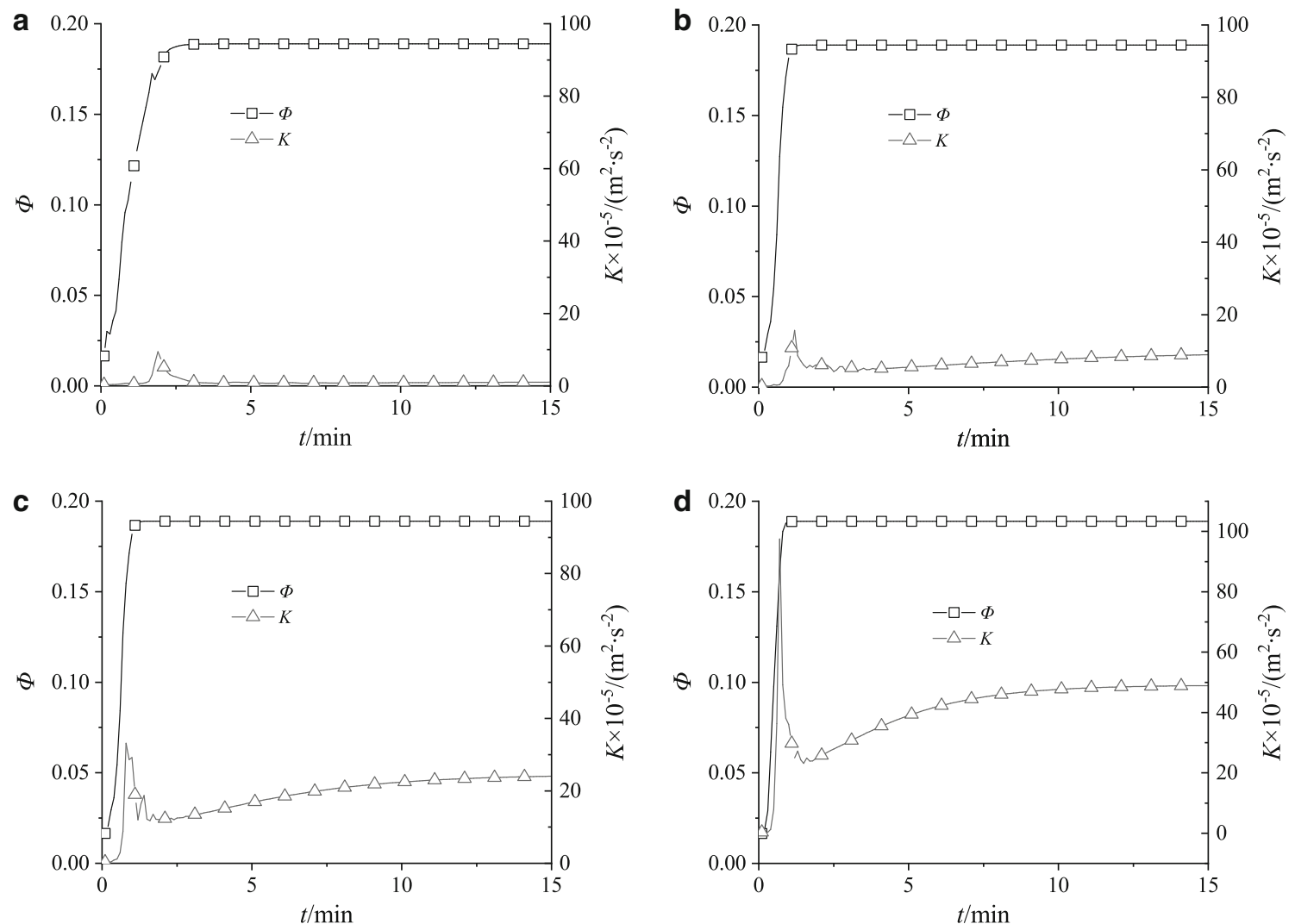

Fig. 8 Relationship between average volume fraction, average turbulent kinetic energy, and time

considerably higher than that of dissolved phosphorus. Considering the characteristics of phosphorus adsorption and desorption, phosphorus adsorbed on sediment particles could be rapidly desorbed and released post sediment resuspension. In practice, the amount of phosphorus in the overlying water significantly increases, resulting in the secondary pollution of water. Figure 9 shows the relationship of the average total phosphorus concentration $C$ and time in Cases 1 and 6 for Sand1 and Sand2 with different adsorption charac- teristics. As could be observed, $C$ varied over time. Given different velocities, $C$ attained equilibrium in a short duration ( $<3 \mathrm{~min}$ ). Additionally, at a faster $\boldsymbol{U}$, the time taken for $C$ to attain equilibrium was shorter. In both cases, the value of $\boldsymbol{U}$ increased by nearly 15.6 times, whereas $C$ increased only slightly, by $\sim 4.4 \%$ and $\sim 4.8 \%$, in the water of Sand 1 and Sand2. However, because of the different adsorption characteristics of the two sediment samples, the difference in $C$ in the water exceeded $37 \%$. Post sediment resuspension, $\boldsymbol{U}$ 


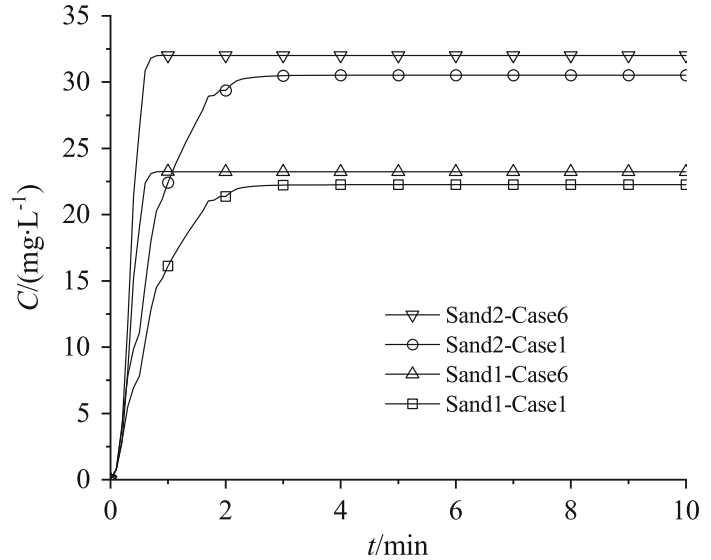

Fig. 9 Relationship between average total phosphorus concentration and time

changed the time taken by $C$ for attaining equilibrium, without significantly increasing its value. Notably, the adsorption and desorption characteristics of sand were an important factor that affected the amount of $C$ in the water.

Because the physical adsorption and desorption of phosphorus on sediment are extremely fast and almost instantaneous, the effect of the dynamics of adsorption and desorption can be ignored. Phosphorus adsorption is generally maximum in the initial time $Q_{\mathrm{m}}$. Equation (1) evidences that the desorption of phosphorus can be regarded as the difference between $Q_{\mathrm{m}}$ and $Q_{\mathrm{a}}$; thus, the desorbed phosphorus concentration $C_{1}$ can be obtained in terms of $\Phi$ and sediment density: $\mathrm{d} C_{1}=\rho_{2} \times \Delta Q \times \mathrm{d} \Phi$. Figure 10 illustrates the relationship between $C_{1}$ and $C$ at different velocities, with Sand1 and Sand2 having different adsorption characteristics. The $Q_{\mathrm{m}}$ of Sand1 was 1.67 times that of Sand2, but no significant difference was observed in $C_{1} / C$ between the two sediments. After the resuspension of different sediments,

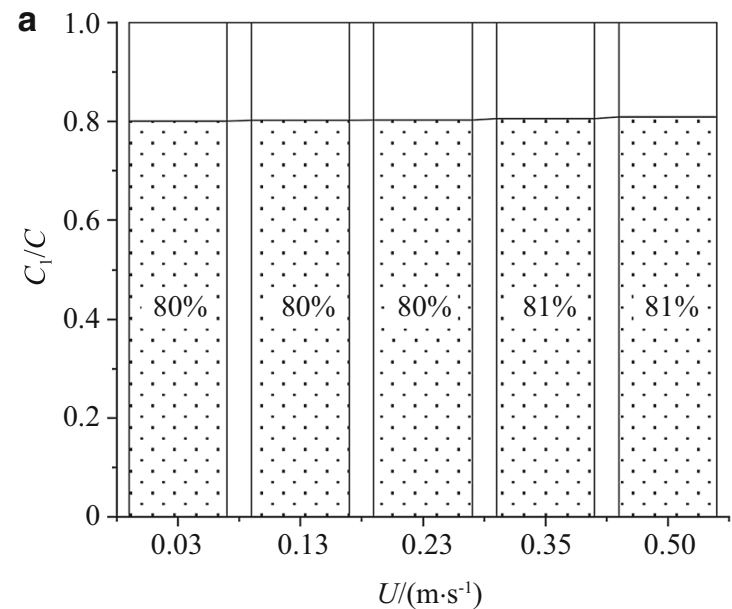

the value of $C_{1}$ was $>80 \%$ of $C$, whereas the concentration of the dissolved phosphorus was $<20 \%$, which is consistent with the monitoring results. Further, $C_{1} / C$ slightly increased with increasing velocity, which suggests that the amount of $C$ mainly depends on $Q_{\mathrm{m}}$ and $\Phi$, i.e., the adsorption-desorption characteristics and water content. Herein, the water content of the two sediments was $57.5 \%$. As the water content was increased to nearly $75 \%, C_{1}$ could be close to the concentration of the dissolved phosphorus. Typically, the value of $C_{1}$ gradually decreases with increasing water content.

Subsequently, the effect of hydrodynamic conditions on $C_{1}$ and $C$ were analyzed post sediment resuspension by comparing the data of Sand1 and Sand2 in Cases 1, 3, 4, and 5. Figure 11 shows the variation of $C$ and $\mathrm{d} C_{1} / \mathrm{d} t$ over time in the sediment samples. As shown, the value of $C$ in the overlying water could attain equilibrium in a short duration under different velocities. Note that the amount of $C$ is closely related to the sediment properties, and is less affected by the velocity. Accordingly, the change in $\mathrm{d} C_{1} / \mathrm{d} t$ in both sediments were similar. A faster $\boldsymbol{U}$ could accelerate the time taken by $C$ for attaining equilibrium while increasing the peak value of $\mathrm{d} C_{1} / \mathrm{d} t$. As soon as $C$ attained equilibrium, $\mathrm{d} C_{1} / \mathrm{d} t$ approximated to 0 . In reference to Fig. 8 , the change in $\mathrm{d} C_{1} / \mathrm{d} t$ could basically be treated the same as that observed in $K$. Thus, a higher $K$ would permit the entrance of more particles in water in unit time, which elevates the value of $\mathrm{d} C_{1} / \mathrm{d} t$. In all cases in Fig. 11, an increase in the value of $\boldsymbol{U}$ was corresponded by twice an increase in the peak value of $\mathrm{d} C_{1} / \mathrm{d} t$ and a reduction of about 2.3 times in the desorption release time. In general, the water content of the sediment in rivers or lakes is $<75 \%$, i.e., the phosphorus pollutants are primarily sourced from the desorption release of the sediment post resuspension. With increasing $\boldsymbol{U}$, the sediment particles in the water increase, producing a sharp increase in the value of $C$ in the overlying water, which reaches a peak shortly. Note that the phospho-

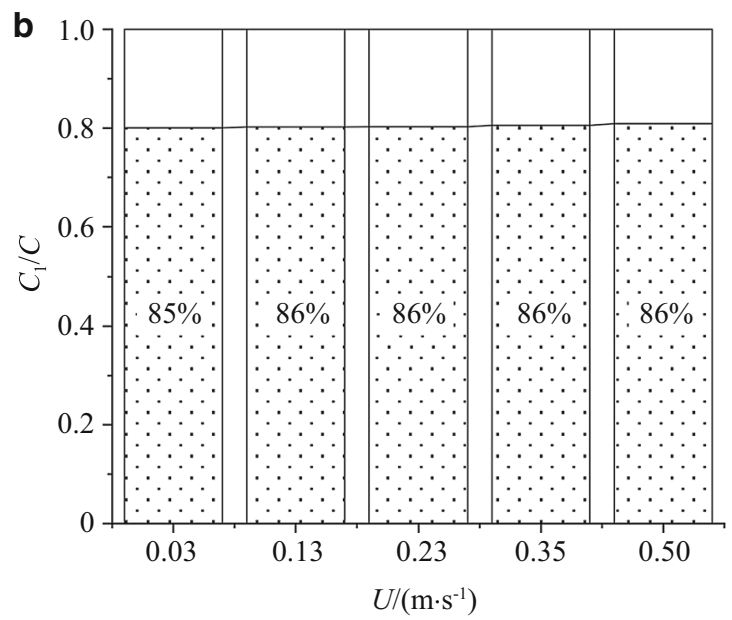

Fig. 10 Bar graph of $C_{1} / C$ at different velocities 

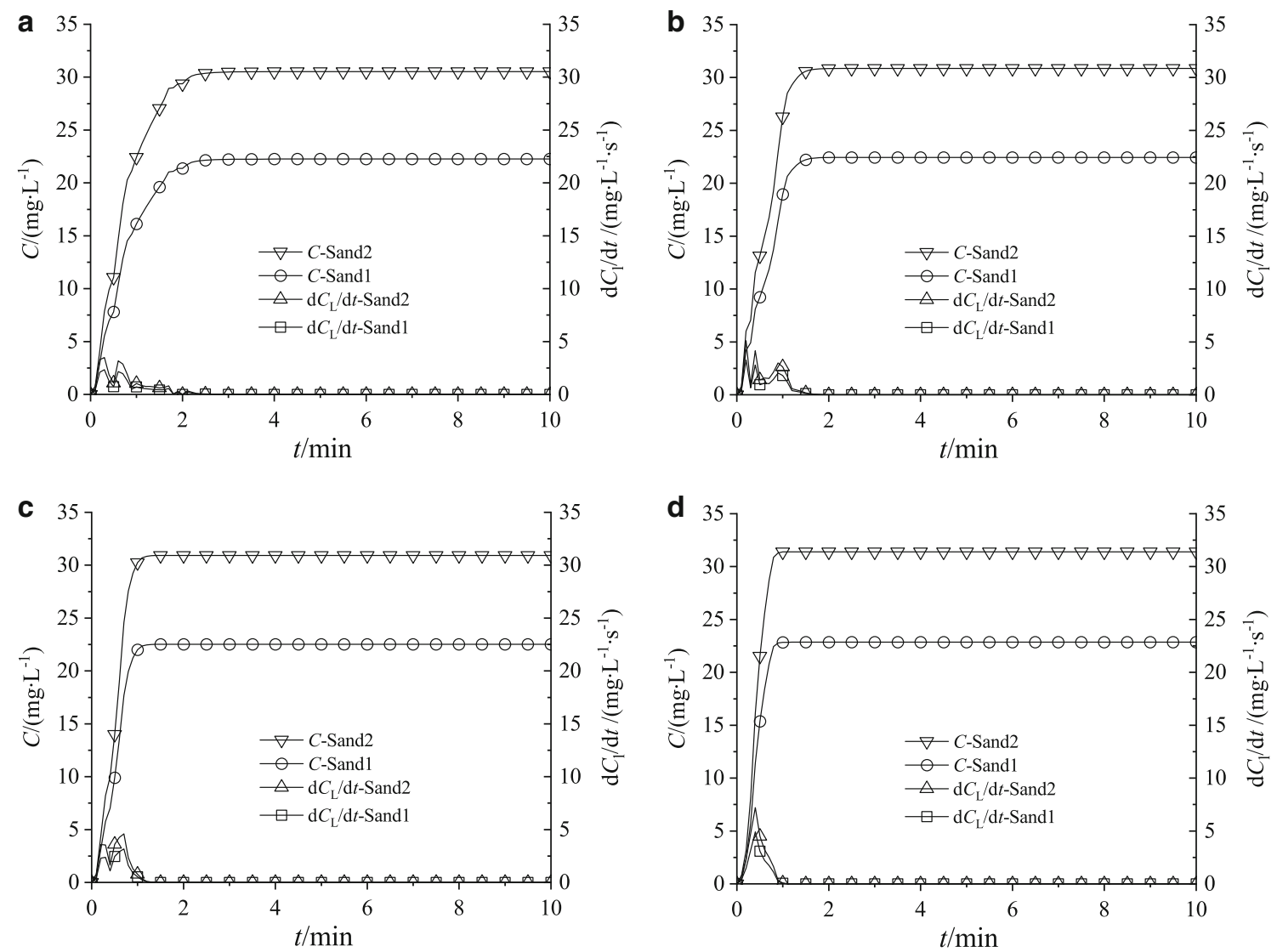

Fig. 11 Relationship of average total $\mathrm{P}$ concentration, $\mathrm{d} C_{\mathrm{l}} / \mathrm{d} t$ and, time

rus release is characterized by a concentrated and massive release. Post sediment resuspension, both the hydrodynamic conditions and sediment properties play an important role in the release process, enabling the total phosphorus concentration to change over a short duration, which consequently affects the water environment.

\section{Conclusion}

The strong affinity between phosphorus and sediment causes majority of the phosphorus in the water to be adsorbed on the surface of the sediment particles. Comparatively, the concentration of the adsorbed phosphorus is much higher than that of dissolved phosphorus. Phosphorus enters the water through desorption post sediment resuspension and becomes a secondary water pollution source.

The water content of the sediment in rivers or lakes is generally less than $75 \%$, implying that the phosphorus pollutants mainly originate from the desorption release from the sediment. Herein, the desorbed phosphorus concentration $C_{1}$ in Sand1 and Sand 2 reached more than $80 \%$ of the total phosphorus concentration $C$. The desorption release $\mathrm{d} C_{1} / \mathrm{d} t$ was consistent with the change in $K$, i.e., with higher $K$,

more, particles enter the water in unit time, yielding a greater $\mathrm{d} C_{1} / \mathrm{d} t$. Further, with increasing $\boldsymbol{U}, C$ rapidly increases in the overlying water and reach a peak value in a short duration. Specifically, the time taken by $C$ to reach its peak value is closely related to the characteristics of the flow field (i.e., velocity and turbulent kinetic energy), whereas its value is closely related to the sediment properties (i.e., maximum adsorption capacity, adsorption strength, and water content).

The coupling mechanism of the overlying water, sediment, and pollutant is important in the phosphorus release from sediment resuspension. Thus, herein, the coupled mechanical model was used to establish and elucidate the relationships among flow field characteristics, sediment resuspension, and pollutant concentration, and to further explain the phosphorus release process from the sediment to the overlying water. Post sediment resuspension, the hydrodynamic conditions and sediment properties become crucial to phosphorus release. The time taken by the total phosphorus concentration for reaching the peak value is closely related to the hydrodynamic conditions, whereas the amount of phosphorus is closely related to the sediment properties. Additionally, the phosphorus release is a concentrated and massive release, which can impact the water environment in a short duration. Understanding this coupling mechanism in greater depth is 
significant for protecting the water environment and in preventing and controlling water pollution.

Acknowledgements This work was financially supported by the Strategic Priority Research Program of the National Key R\&D Program of China (Grants 2018YFC1505500 and 2018YFC1505504), the Chinese Academy of Science (Grant XDB10030303), and the National Natural Science Foundation of China (NSFC) (Grants 11802313 and 11872117).

\section{References}

1. Zhang, C., Yu, Z.-G., Zeng, G.-M., et al.: Effects of sediment geochemical properties on heavy metal bioavailability. Environ. Int. 73, 270-281 (2014)

2. Wang, D.-Z., Lin, W.-Q.: Study of flow augmentation and water environment rehabilitation for Suzhou Greek. Mech. Eng. 27, 1-12 (2005)

3. Lin, W.-Q., Lu, S.-Q., Chen, Y.-Z.: An application of ecodynamic model in evaluating eutrophication control measures for Dianshan Lake in Shanghai. Shanghai Environ. Sci. 29, 1-10 (2010)

4. Yu, X.-Z., Zhong, D.-Y., Li, J.-X.: Review of studies on sediment in water environment. J. Sedim. Res. 6, 75-81 (2004)

5. Cheng, P.-D., Zhu, H.-W., Wang, D.-Z., et al.: Numerical research for contaminant release from un-suspended bottom sediment under different hydrodynamic conditions. J. Hydrodyn. 25, 620-627 (2013)

6. Cheng, P.-D., Zhu, H.-W., Wang, D.-Z., et al.: Sediment rarefaction resuspension and contaminant release under tidal currents. J. Hydrodynam. 26, 827-834 (2014)

7. Chen, M.-H.: The phosphorus adsorption rule and surface microtopography change of sediment particle. Ph.D. Thesis, Tsinghua University, Beijing (2008).

8. Zhu, G.-W., Gao, G., Qin, B.-Q.: Geochemical characteristic of phosphorus in sediment of a large shallow lake. Adv. Water Sci. 14, 714-719 (2003)

9. Cheng, P.-D., Zhu, H.-W., Wang, D.-Z., et al.: Transport mechanisms of contaminants released from fine sediment in rivers. Acta. Mech. Sin. 31, 791-798 (2015)

10. Li, B., Zhang, K., Zhong, B.-C., et al.: An experimental study on release of pollutants from sediment under hydrodynamic conditions. Chin. J. Hydrodynam. 23, 126-133 (2008)

11. Zhu, H.-W., Zhang, K., Wang, D.-Z., et al.: Effects of particles and pore water in release of pollutants due to sediment resuspension. J. Hydrodynam. 26, 631-641 (2011)

12. Zhu, H.-W., Cheng, P.-D., Li, W., et al.: Empirical model for estimating vertical concentration profiles of re-suspended, sedimentassociated contaminants. Acta. Mech. Sin. 33, 846-854 (2017)

13. Zhang, L., Zhong, D.-Y., Wu, B.-S., et al.: The convectiondispersion equation and the mechanism of suspension in turbulent open-channels. Chin. J. Theor. Appl. Mech. 45, 83-93 (2013)

14. Bai, J., Fang, H.-W., He, G.-J., et al.: Numerical simulation of erosion and transport of fine sediments by large eddy simulation. Chin. J. Theor. Appl. Mech. 49, 65-74 (2017)

15. Fetters, K.J., Costello, D.M., Hammerschmidt, C.R., et al.: Toxicological effects of short-term resuspension of metal-contaminated freshwater and marine sediments. Environ. Toxicol. Chem. 35, 676-686 (2016)
16. Matisoff, G., Watson, S.B., Guo, J., et al.: Sediment and nutrient distribution and resuspension in Lake Winnipeg. Sci. Total Environ. 575, 173-186 (2017)

17. Lepage, H., Launay, M., Coz, J.L., et al.: Impact of dam flushing operations on sediment dynamics and quality in the upper Rhône River France. J. Environ. Manag. 225, 109886 (2020)

18. Fang, H., Huang, L., Wang, J., et al.: Environmental assessment of heavy metal transport and transformation in the Hangzhou Bay China. J. Hazard. Mater. 302, 447-457 (2016)

19. Ministry of Environmental Protection of the People's Republic of China. Water Quality-Determination of Orthophosphate and Total Phosphorus-Continuous Flow Analysis (CFA) and ammonium Molybdate Spectrophotometry (HJ 670-2013). China Environmental Science Press, Beijing, China (2013).

20. Stickel, J.J., Powell, R.L.: Fluid mechanics and rheology of dense suspensions. Annu. Rev. Fluid Mech. 37, 129-149 (2005)

21. Hinch, E.J.: The measurement of suspension rheology. J. Fluid Mech. 686, 1-4 (2011)

22. Launder, B.E., Spalding, D.B.: The numerical computation of turbulent flows. Comput. Methods Appl. Mech. Eng. 3, 269-289 (1974)

23. Wilcox, D.C.: Turbulence Modeling for CFD, 3rd edn. DCW Industries Inc., La Canada Flintridge (2010)

24. Maron, S.H., Pierce, P.E.: Application of ree-eyring generalized flow theory to suspensions of spherical particles. J. Colloid Sci. 11, 80-95 (1956)

25. Quemada, D.: Rheology of concentrated disperse systems and minimum energy dissipation principle. Rheol. Acta 16, 82-94 (1977)

26. Mendoza, C.I., Santamaria-Holek, I.: The rheology of hard sphere suspensions at arbitrary volume fractions: An improved differential viscosity model. J. Chem. Phys. 130, 044904 (2009)

27. Shewan, H.M., Stokes, J.R.: Analytically predicting the viscosity of hard sphere suspensions from the particle size distribution. J. Nonnewton. Fluid Mech. 222, 72-81 (2015)

28. Zhang, B.-Z.: The finite element method in fluid dynamics. China Machine Press, Beijing (1984)

29. Ignat, L., Pelletier, D., Ilinca, F.: A universal formulation of twoequation models for adaptive computation of turbulent flows. Comput. Methods Appl. Mech. Eng. 189, 1119-1139 (2000)

30. Hughes T, Franca LP, Hulbert GM. A new finite element formulation for computational fluid dynamics: VIII The galerkin/leastsquares method for advective-diffusive equations. Comput. Methods Appl. Mech. Eng. 73, 173-189 (1989).

31. Elman, H.C., Silvester, D.J., Wathen, A.J.: Finite elements and fast iterative solvers: with applications in incompressible fluid dynamics. Oxford University Press, New York (2005)

32. Cotter, C.J., Ham, D.A., Pain, C.C., et al.: LBB stability of a mixed Galerkin finite element pair for fluid flow simulations. J. Comput. Phys. 228, 336-348 (2009)

33. Chorin, A.J.: Numerical solution of the Navier-Stokes equations. Math. Comput. 22, 745-762 (1968)

34. Gresho, P.M., Sani, R.L.: Incompressible flow and the finite element method Isothermal Laminar Flow, vol. 2. Wiley, Chichester (2000) 